\title{
Pure Condition of Both 6R Serial and 3-PRS Parallel Robots Using Grassmann-Cayley Algebra
}

\author{
Luc Djimon Clément Akonde ${ }^{1 *}$, Alain Adomou², Tognon Clotilde Guidi² \\ ${ }^{1}$ Département du Génie Industriel et Maintenance (GIM), Institut Universitaire de Technologie (IUT) de Lokossa, Université \\ Nationale des Sciences, Technologies, Ingénierie et Mathématiques (UNSTIM), d’Abomey, Benin \\ ${ }^{2}$ Institut Universitaire de Technologie (IUT) de Lokossa, Université Nationale des Sciences, Technologies, Ingénierie et \\ Mathématiques (UNSTIM), d'Abomey, Benin \\ Email: *goering20@yahoo.fr
}

How to cite this paper: Akonde, L.D.C., Adomou, A. and Guidi, T.C. (2018) Pure Condition of Both 6R Serial and 3-PRS Parallel Robots Using Grassmann-Cayley Algebra. Modern Mechanical Engineering, 8, 233-248.

https://doi.org/10.4236/mme.2018.84016

Received: August 19, 2018

Accepted: November 6, 2018

Published: November 9, 2018

Copyright (c) 2018 by authors and Scientific Research Publishing Inc. This work is licensed under the Creative Commons Attribution International License (CC BY 4.0).

http://creativecommons.org/licenses/by/4.0/

\begin{abstract}
This research presents Pure Condition approach, which has used in analyzing simultaneously the singularity configuration and the rigidity of mechanism. The study cases analysis is implemented on variable joints orientation of $6 \mathrm{R}$ (Revolute) Serial Manipulators (SMs) and variable actuated joints position of 3-PRS (Prismatic-Revolute-Spherical) Parallel Manipulators (PMs) using Grassmann-Cayley Algebra (GCA). In this work we require in Projective Space both Twist System (TS) and Global Wrench System (GWS) respectively for serial and parallel manipulators which represent the Jacobian Matrix (J) in symbolic approach to Plücker coordinate vector of lines and unify framework on static and kinematics respectively. This paper, works, is designed to determine geometrically at symbolic level the vanished points of inverse form of this Jacobian Matrix (J) which called superbracket in GCA. The investigation vary to those reported early by introducing GCA approach on the singularity of serial robot, variable joints orientation and actuated positions on robot manipulators (RMs) to analyze rigidity frame work and singularity configuration which involve simultaneously Pure Condition. And the results also revealed a single singularity condition which contains all particulars cases and three general cases such as the shoulder, elbow and wrist singularity for SMs while double, single and undermined singularities respectively for 3-PRS, 3-PRS and 3-PR $\underline{S}$ PMs which contain all generals and particulars cases.
\end{abstract}

\section{Keywords}

Pure Condition, 6R SMs, 3-PRS PMs, Superbrackets, GCA 


\section{Introduction}

Pure Condition is a scalar value that is needed to identify both singularity configuration and rigidity of robot framework. Singularity of Serial robot arises when certain direction is unattainable and one or more Degree of Freedom (DOF) is loosed while the end-effector of Parallel robot becomes uncontrollable and gained more DOF. Rigidity of Robot framework preserves the length bar-joint which is lacked at singularity [1]. One classical approach to solve Pure Condition problem is determining where J is non-singular. In the past years, extensive studies were carried out on analytically [2], algebraically [3] [4], kinematical [5] [6], numerical [5] [7], geometrical [2] [8] and simulation [9] approaches. Since Jacobian Matrix J is expressed in Plücker coordinate vector of lines [10] [11], recently experiments by S. Amine, S. Caro et al. [12] have suggested the geometric method associated with dependency of Plücker vector lines to get the determinant of Matrix J which is formulated in GCA language [10] [13]. On the basis of previous literature data, we intend to investigate pure condition of $6 \mathrm{R}$ SMs within variable orientated joints which TS is calculated before computing its superbracket while 3-PRS PMs within variable actuated joints: 3-PRS; 3-PRS and 3-PR $\underline{S}$ which GWS are calculated before computing their superbrackets decomposition. Interpretation of the vanishing condition of these superbrackets decomposition involves the robot pure condition.

The key contribution in this paper is a simultaneous determination of both singularity condition of Robots Manipulators and rigidity framework without algebraic calculus by Grassmann-Cayley Algebra approach. We shall calculate the determinant of the Jacobian Matrices in coordinate free manner by developing and reducing the Superbracket expression. A novelty from other research is that Serial Robot in this paper is cheeked and investigated by Grassmann-Cayley Algebra.

This paper is organized as follows: Section 2 recalls mathematics background of projective space such as Plücker coordinate of vectors line, screw theory, twist system, Global Wrench System with their associate graphs before the concept of brackets which represent the Jacobian matrix used in GCA applied to robot manipulators. Section3 describes respectively 6R SMs and 3-PRS PMs with their adopted representation. Section 4 focuses firstly on computing the TS of $6 \mathrm{R}$ SMs where all joints are simultaneous actuated and the GWS of 3-PRS PMs where the actuators are sequenced on prismatic, revolute or spherical joint. Secondly on the vanished condition of TS and GWS which represent the determinant of matrix J in GCA language. The interpretation, comparison and verification of these obtained results are discussed as the pure condition of robot. Finally, concluding and remarks are given in Section 5.

\section{Mathematics of Robot Manipulator Using Grassmann-Cayley Algebra}

This section gives firstly a mathematical background of both TS and GWS from Plücker coordinate line in Projective Space; useful tool to describe the instan- 
taneous motion of robot manipulators (RMs). Secondly brief notion of GCA is applied on robot manipulators to determine the Jacobian Matrix in bracket form. More details on GCA and superbrackets decomposition should refer to [10]-[17].

First of all, the brief background information about this research is provided in the Diagram 1 below.

\subsection{Projective Space Extended to Robot Motion}

The projective space refers to the affine space augmented by the points at infinity where any pair of parallel lines can be said to meet at the unique point at infinity on the plane $\Pi_{\infty}$. In projective space any point (line) has four (six) homogeneous coordinates and each line is called Plücker line which can be extended to coordinates for the screws and the duality twist-wrenches in application for kinematics of robot manipulators [12]. A finite line, $L$, is represented by its Plücker coordinates vector $F$ :

$$
F=(s ; r \times s)
$$

where $s$ is the line direction, $(r \times s)$ represents the moment of $L$ with respect to the origin and $r$ is the position vector of any point on $L$. An instantaneous screw axis is a Plücker coordinate vector line with it associated pitch in a given position, then the screw axis of PMs is described by:

$$
\hat{\$}=\left[s ;\left(s_{0} \times s\right)+h s\right]^{T}
$$

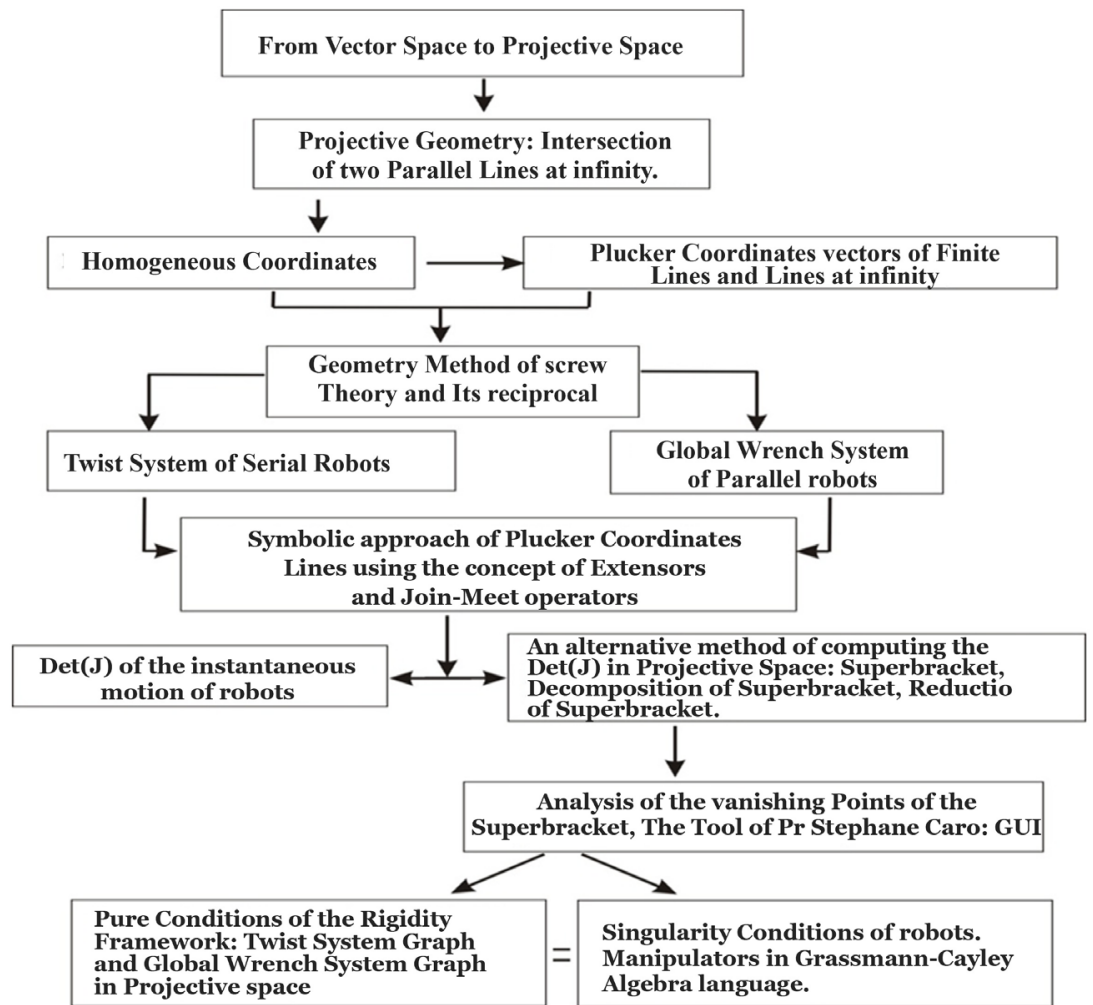

Diagram 1. Brief background information about this research. 
with, $s$ the unit vector along the screw axis, $s_{0}$ the position vector of a point on the screw axis with respect to a reference frame, $h$ the pitch of the screw. A zero pitch screw $\hat{\$}_{0}$ and an infinite pitch screw $\hat{\$}_{\infty}$ can be respectively identified with a Plücker coordinate vectors of a finite line and a line at infinity:

$$
\begin{gathered}
\hat{\$}_{0}=\left[s ;\left(s_{0} \times s\right)\right]^{T} \\
\hat{\$}_{\infty}=[0 ; s]^{T}
\end{gathered}
$$

Since $n$ number of independents kinematic chains form $(n)$ screw space which is composed of $(n)$ system screws, all screws which are reciprocal to a $(n)$ system screws form (6-n)system. Two zero pitches screws, $\hat{\$}_{0}$ and $\hat{\$}_{0}^{\prime}$, are reciprocal to each other if and only if their axes are coplanar. A zero pitch screw $\hat{\$}_{0}$ is reciprocal to an infinite pitch screw $\hat{\$}_{\infty}$ if only if their directions are orthogonal to each other. Two infinite pitch screws $\hat{\$}_{\infty}$ and $\hat{\$}_{\infty}^{\prime}$ are always reciprocal to each other. A body instantaneously undergoing a pure rotation about an axis $l$ is a twist of zero pitch $\hat{\$}_{0}$. A body instantaneously undergoing a pure translation along an axis $l$ is a twist of infinite pitch $\hat{\$}_{\infty}$. A manipulator subjects to a pure force along the axis $l$ is a wrench of zero pitch screw $\hat{\$}_{0}$. A body subject to a pure couple is a wrench of infinite pitch screw $\hat{\$}_{\infty}$. In robotics; wrench is a screw representing a combination of a force and a couple acting on a manipulator [12].

\subsection{Twist System (TS) and Global Wrench System (GWS)}

Twist Space of a serial kinematic chain is the support of the join extensors that represents the twist spaces, and then the Twist System $T^{i}$ of a serial kinematic chain composed of $k$ joints extensors is described as [17]:

$$
T^{i}=T^{1} \vee T^{2} \vee \cdots \vee T^{k}
$$

Similarly, the Wrench Space of a parallel kinematic chain is the support of the join extensors that represents the wrench spaces and then the Wrench System $W_{n}$ of an $n$ parallel kinematic chain composed of $m$ serial chains is described as [17]

$$
W_{i}=W_{1} \vee W_{2} \vee \cdots \vee W_{n}
$$

Each actuated serial limb constraint the mobility of mechanism. The constraint wrench system $\left(F_{i}\right)$ of limb $l_{i}$ is obtained as a reciprocal screw system to all joints screws of the corresponding limb $l_{i}$ and it's imposed by architecture of the robot while the actuation wrench system $\left(W_{n}\right)$, imposed by the actuator of $l_{i}$ is obtained by locking the actuator of $l_{i}$ before determining a reciprocal screw to the joint screw except that of the actuated $\operatorname{link}_{n}$. The combination of $\left(F_{i}\right)$ which defines how the mobile platform (end-effector) is forced by the limbs and $\left(W_{n}\right)$ which defines how the actuators act on dof of the effector, represents the instantaneous motion of robot manipulator or Jacobian matrix of Plücker lines also called $G W S$; a combinatory platform of these wrenches system 
[18] and described as:

$$
G W S=\left(W_{n}, F_{i}\right)=\left(\hat{\$}_{a}^{1}, \cdots, \hat{\$}_{a}^{n}, \hat{\$}_{c}^{1}, \cdots, \hat{\$}_{c}^{6-n}\right)
$$

with $W_{n}=\underbrace{\hat{\$}_{a}^{1}, \cdots, \hat{\$}_{a}^{n}}_{(n) \text { system-actuated }}$ and $F_{i}=\underbrace{\hat{\$}_{c}^{1}, \cdots, \hat{\$}_{c}^{6-n}}_{(6-n) \text { system-constraint }}$. The $\operatorname{det}(J)$ is written in the $G W S$ form as:

$$
\operatorname{det}(J)=[G W S]=[\underbrace{W_{1}, \cdots, W_{n}}_{(n) \text { system }}, \underbrace{F_{1}, \cdots, F_{i}}_{(6-n) \text { system }}]
$$

The bracket of these vectors is defined as the determinant of matrix having $w_{i}$ as its columns and described as:

$$
\left[w_{1}, w_{2}, \cdots, w_{k}\right]=\operatorname{det}\left|\begin{array}{cccc}
x_{1,1} & x_{1,2} & \cdots & x_{1, k} \\
\vdots & \vdots & \ddots & \vdots \\
x_{k, 1} & x_{k, 2} & \cdots & x_{k, k}
\end{array}\right|
$$

The determinant GCA in language represents the symbolic approach of Plücker coordinate lines and is used to analyze the pure condition without algebraic coordinates. In consequence, the system is linearly dependent when:

$$
w_{1} \vee w_{2} \vee \cdots \vee w_{k}=\left[w_{1}, w_{2}, \cdots, w_{k}\right]=0
$$

\section{Description and Adopted Representation of both 6R SMs and 3-PRS PMs}

\subsection{Description and Adopted Representation of 6R SMs}

The 6R SMs consist of seven links $l_{i(i=1,2, \cdots, 7)}$ connected in succession by six revolute joints (Figure 1). The link $l_{i}$ fixed to the ground in a fixe frame $O\left(x_{1} y_{1} z_{1}\right)$ while the last three non-coplanar axis intersect at a unique common point $h$. The revolute axis $Z_{i}$ are respectively the joint axis of $I_{i}$ and $I_{i+1}$ where $i=1,2, \cdots, 6 ; Z_{1}$ is perpendicular to both $Z_{2}$ and $Z_{3}, Z_{2}$ and $Z_{3}$ are parallel. $Z_{4}, Z_{5}$ and $Z_{6}$ are three no-coplanar intersecting axis at a unique point $h$ (Figure 2). In projective space, we choose two points on each of these six joint axis centers:

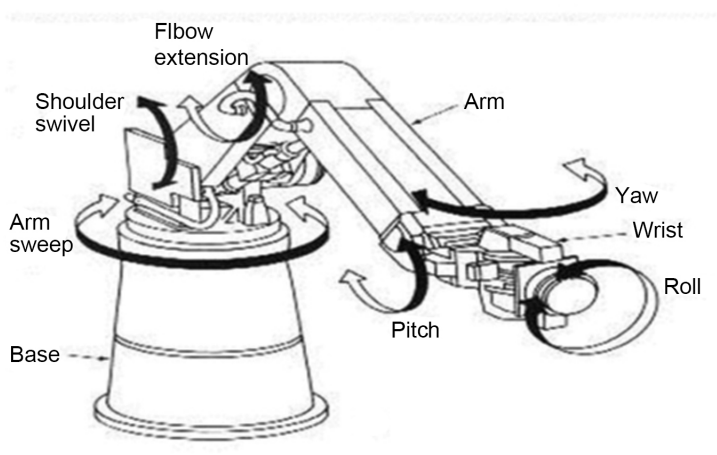

(a)

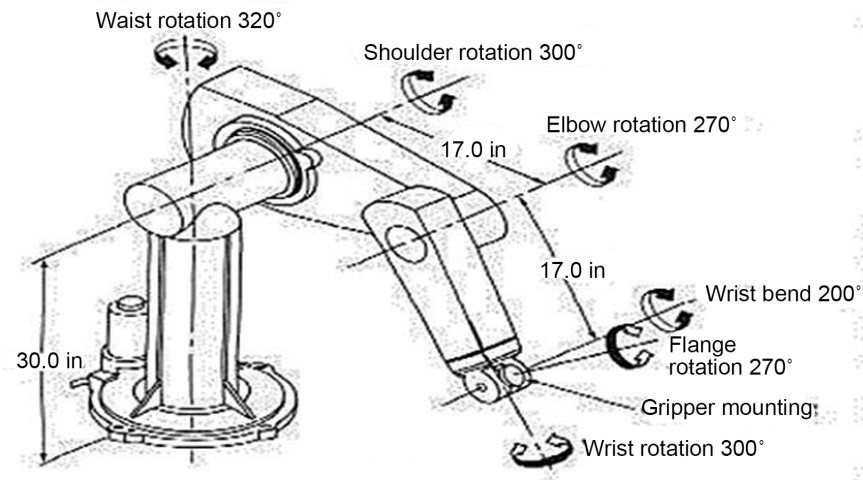

(b)

Figure 1. General 6 revolute wrist serial manipulator for spray painting robot. 


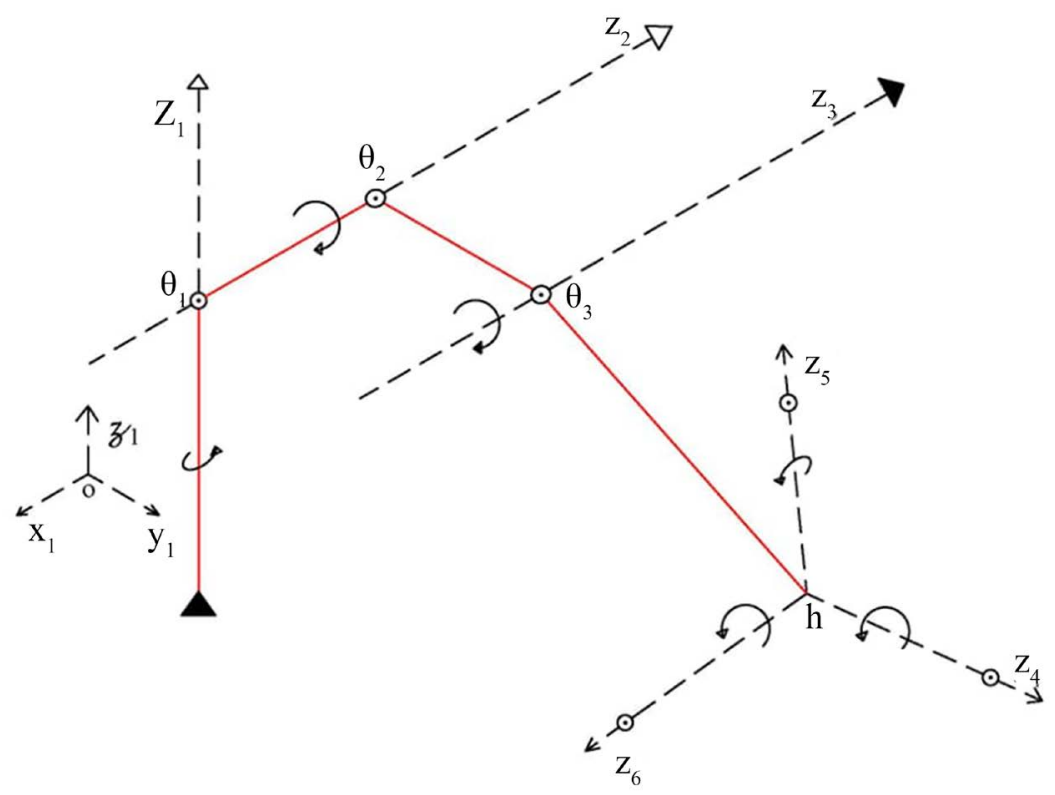

Figure 2. Descriptions of 6RSMs.

$a B, c D, f E, h G, h J$ and $h L$ respectively on $z_{1}, z_{2}, z_{3}, z_{4}, z_{5}$ and $z_{6}$ (Figure 3).

\subsection{Description and Adopted Representation of 3-PRS PMs}

Each independent $l_{i}$ has five degrees of freedom (Figure 4). Express as one translation, one revolute and one spherical joint which is consist of three intersecting and non coplanar rotations joints at $S_{i}\left(u_{i}, v_{i}, w_{i}\right)$ The input of the mechanism consists of three variable positions of the actuated joints defined as: Firstly the prismatic joints are actuated and all other joints are passive. Secondly the revolute joints are actuated and all other joints are passive. Thirdly the spherical joints are actuated and all other joints are passive. The axis $\boldsymbol{p}_{i}$ of the prismatic joint and the following axis $r_{i}$ of the revolute joint are perpendicular. Let $\boldsymbol{\alpha}_{i}$ be the plane formed by the spherical joint center $S_{i}$ and the revolute joint axis $\boldsymbol{r}_{i}$ (Figure 5). Let $\beta_{i}$ be the plane which contains the spherical joint center $S_{i}$ and perpendicular to the prismatic joint $\boldsymbol{p}_{\boldsymbol{i}}$ (Figure 5). Consequently $\boldsymbol{\alpha}_{i}$ and $\boldsymbol{\beta} \boldsymbol{i}$ intersect each over at a line $F_{i}$. Lines $a b$, $e f$ and $i j$ represent respectively $F_{1}, F_{2}$ and $\boldsymbol{F}_{3}$ while $\boldsymbol{c d}, \boldsymbol{g h}$, and $\boldsymbol{k} \boldsymbol{l}$ represent respectively $\boldsymbol{r}_{1}, \boldsymbol{r}_{2}$ and $\boldsymbol{r}_{3}$. Thus each $\boldsymbol{F}_{\boldsymbol{i}}$ parallel to each $r_{\dot{r}}$. Let $\gamma_{i}$ be the plane formed by lines perpendicular to the prismatic joint $\boldsymbol{p}_{\boldsymbol{i}}$ and coplanar to the revolute joint $\boldsymbol{r}_{\boldsymbol{i}}$ (Figure 6).

\section{TS and GWS Computation Respectively for 6R SMs and 3-PRS PMs in Coordinate Free Manner}

\subsection{TS Computation for 6R SMs and Its Vanished Points in GCA Approach without Algebraic Calculus}

6R SM consists of 6 serial joints with six center of the motion, the twist space $T$ of serial connection of kinematic links is identified as the join of all the 6-extensors, 


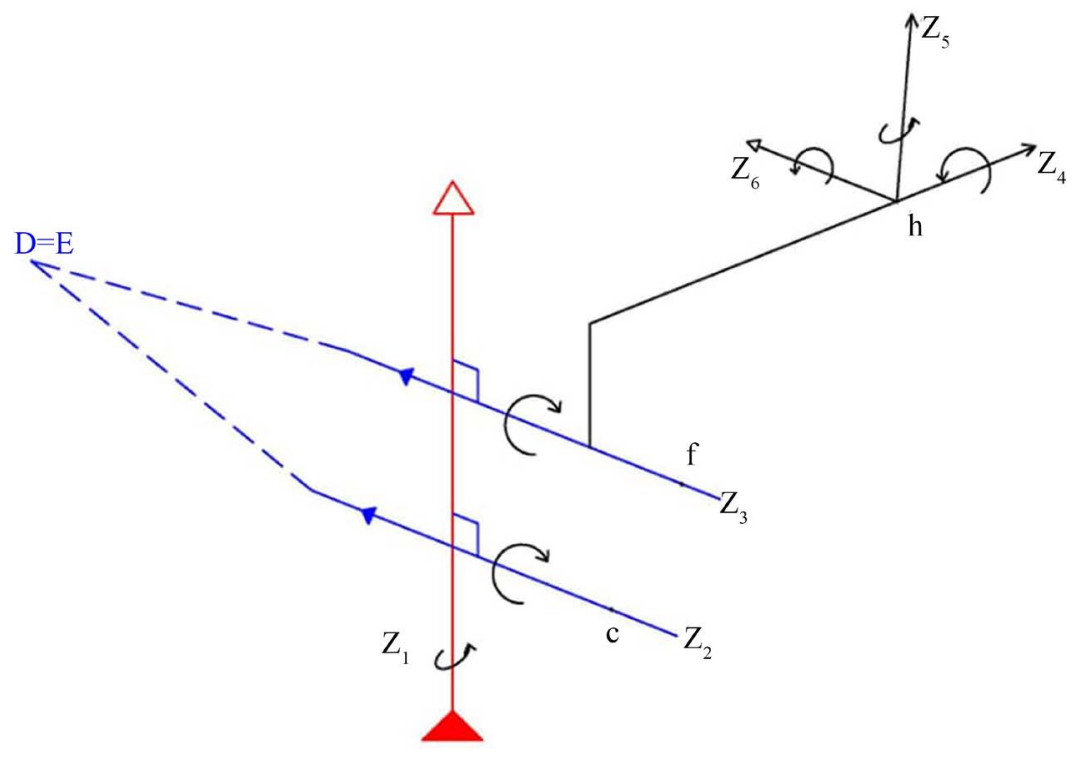

Figure 3. Adopted representation in projective space.

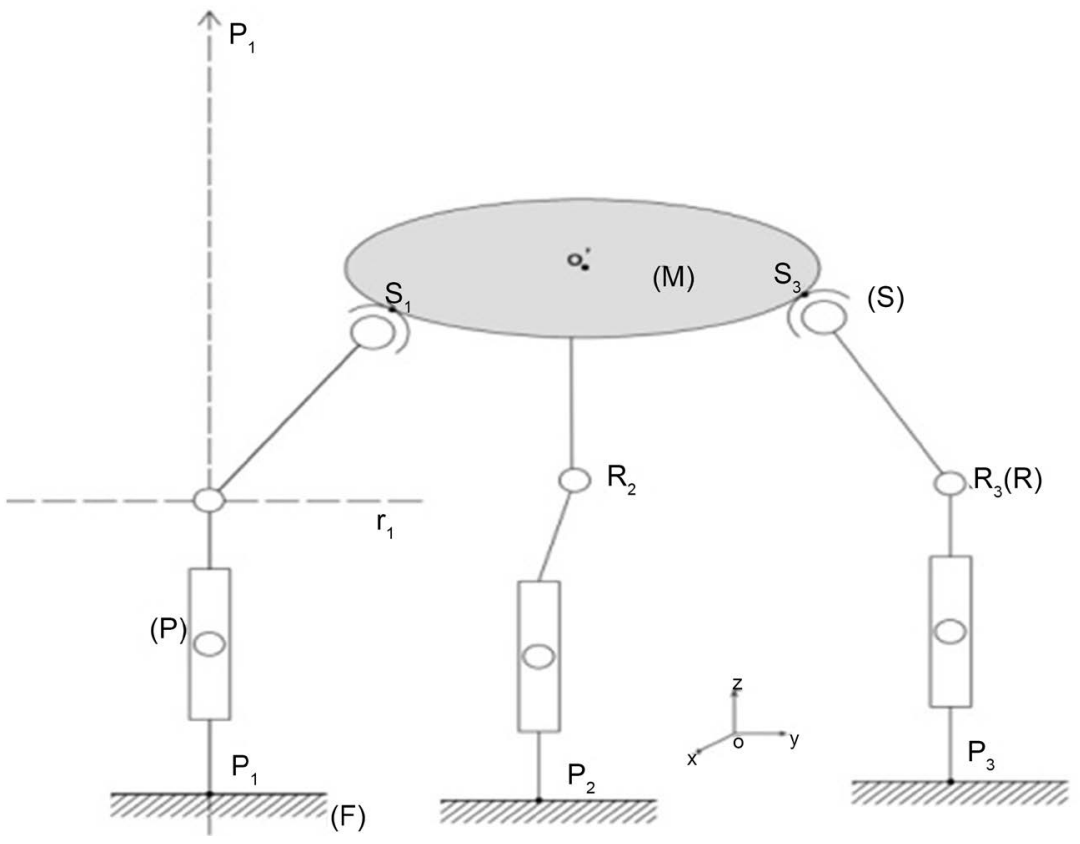

Figure 4. Descriptions of 3-PRS PMs.

see Equation (5). In projective space, any line is formed by two different points which can be either two different finite points or one finite point and one point at infinity. Therefore in this paper, Grassmann-Cayley Algebra applied to 6R serial robot involves the symbolic approach of these six Plücker coordinates finite lines and lines at infinity are graphically represented as its Twist Wrench graph (Figure 7).

Geometrically singularity arises when the joint screws which are Plücker coordinate lines are linearly dependent. $\operatorname{det}\left(J_{t}\right)$ Vanishes then Equation (5) becomes: 


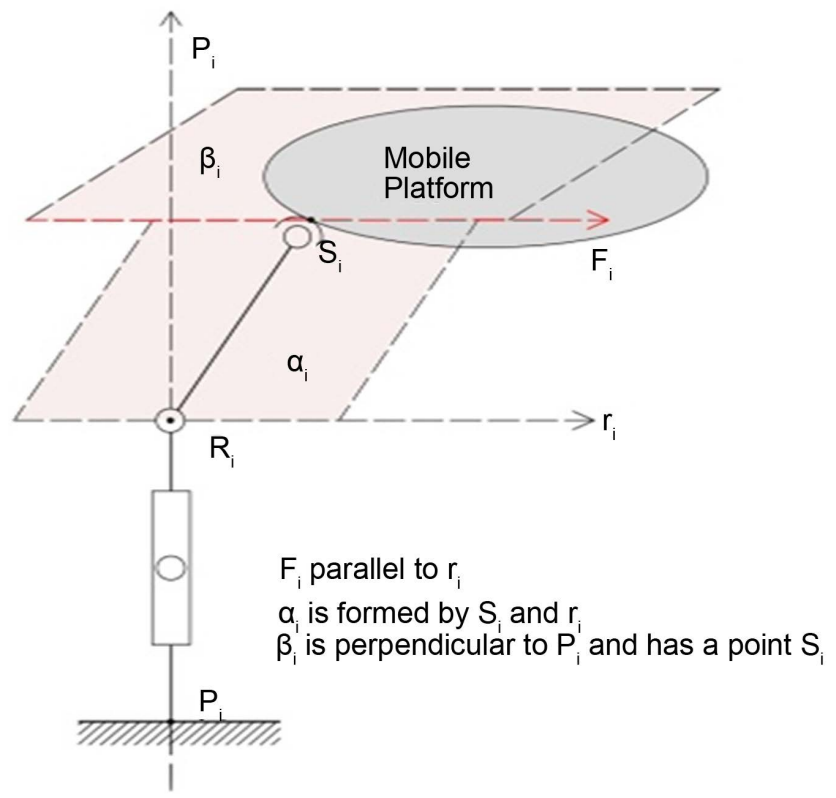

Figure 5. Wenches space $\alpha_{i}$ and $\beta_{i}$

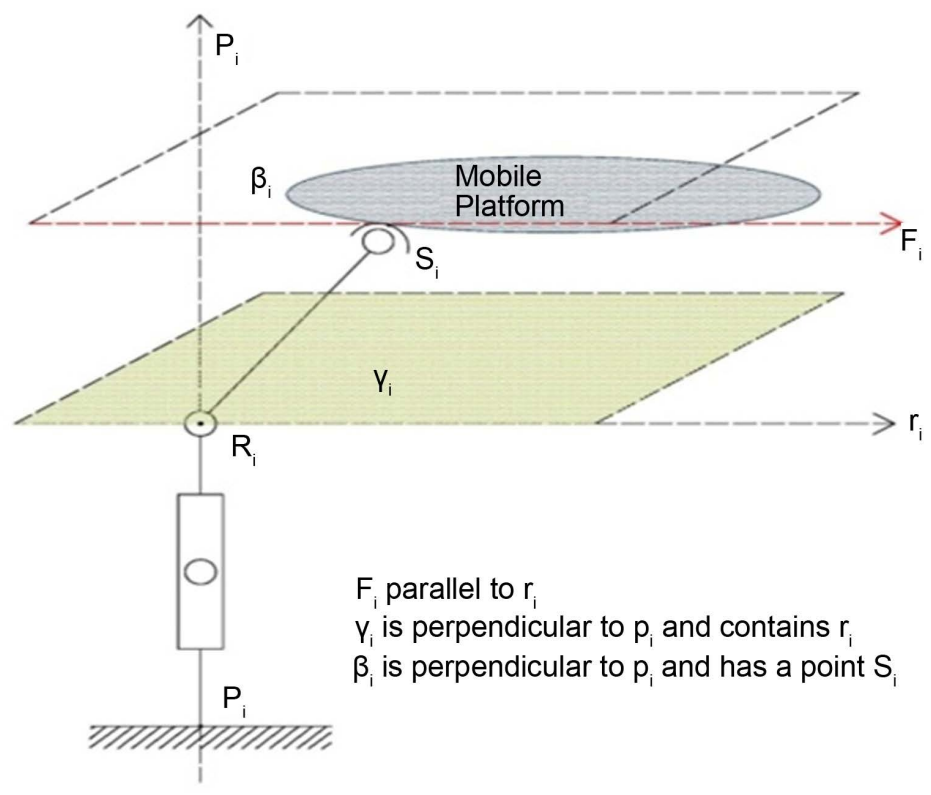

Figure 6. Wenches space $\gamma_{i}$

$$
\begin{aligned}
& T=0 \\
& {[a B, c D, f D, h G, h J, h L]=0}
\end{aligned}
$$

This expression can be developed into 24 combination of linear monomials. Each of them represents the product for three brackets of four projective points. The useful tool, Graphic User Interface, provided by S. Amine, S. Caro et al. [12] perform this calculus and gives the singularity condition:

$$
[a B, c D, f D, h G, h J, h L]=-[a B D h][c f D h][G j h L]
$$




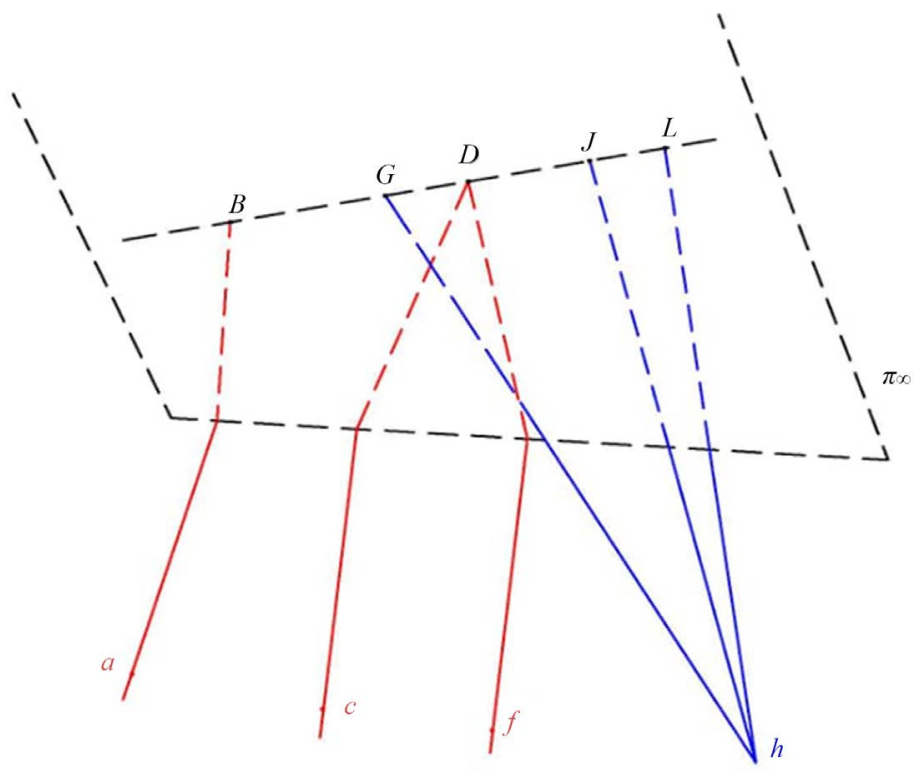

Figure 7. Twist graph of 6R SMs.

And then Equation (12) vanishes when at least one of the three monomials vanish.

If $[a B D h]=0$ then: $a B \wedge h D$ means $h$ lies either on $a B$ or $a B D$. According to the adopted representation $h$ lies on one of: $\left\{z_{1},\left(z_{1} z_{2}\right),\left(z_{1} z_{3}\right)\right\}$ with a particular case $h \in z_{1}$ : a shoulder singularity.

If $[c f D h]=0$ then: $c D \wedge h f$ or $f D \wedge h c$ means $h$ is either collinear to $c D$ or $f D$ since $z_{2}$ and $z_{3}$ are parallel lines and intersect in the projective space at infinity at point $D, h$ must lies on a plane formed by $z_{2} z_{3}$ : it is a an elbow singularity, the end effector is at the boundary of the workspace.

And at last if $[G J h L]=0$ then $h G \wedge L J$ or $h J \wedge L G$ or $h L \wedge G J$, means $z_{4} \wedge L J$ or $z_{5} \wedge L G$ or $z_{6} \wedge G J$, it is obvious that we are in wrist singularity condition and the last three axes $z_{4}, z_{5}, z_{6}$ must be parallel to each over: This condition is impossible to implement practically: except that the wrist design was modified. Two of the last three axis must be parallel to each over.

\subsection{GWS Computation for 3-PRS PMs and Its Vanished Points in GCA Approach without Algebraic Calculus}

Each limb $l_{i}$ of a 3-PRS PMs consist of five serial kinematic chains, a twist $T^{i}$ of each limb $l_{i}$ form 5-system. Instantaneously the composition of these five serial twists corresponds to their simple addition in the projectiv space (Equation (13) [10] [12] [17]

$$
T^{i}=\left[\left(\hat{\$}_{p i}\right)+\left(\hat{\$}_{r i}\right)+\left(\hat{\$}_{u i}\right)+\left(\hat{\$}_{v i}\right)+\left(\hat{\$}_{w i}\right)\right]
$$

with $\hat{\$}_{p i}=\left[0, p_{i}\right]^{T} ; \hat{\$}_{r i}=\left[r_{i},\left(R_{i} \times r_{i}\right)\right] ; \hat{\$}_{u i}=\left[u_{i},\left(S_{i} \times u_{i}\right)\right] ; \hat{\$}_{v i}=\left[v_{i},\left(S_{i} \times v_{i}\right)\right]$; $\hat{\$}_{w i}=\left[w_{i},\left(S_{i} \times w_{i}\right)\right]$ where $p_{i}, r_{i}, u_{i}=S_{i} u_{i}, v_{i}=S_{i} v_{i}$ and $w_{i}=S_{i} w_{i}$ are respectively the unit vectors along the prismatic, revolute and the three intersecting non-coplanar revolute joints axis of the spherical joint for the $i$-th limb (Figure 
4).

In this paper, each serial limb, PRS PMs has five degree of freedom while the closed chain of the 3-PRS fully PMs has only three. This means the limbs constrained the mobile platform by three degree of freedom when each limb is driven by one actuator. The mechanism supposes to be constrained when it is activated and may be stressed at some critical poses.

Constraint wrench system $F_{i}$, the reciprocal constraint wrench system to all (5) system twist $T^{i}$ form a (1)ystem constraint wrench of zero pitch. This constraint wrench system $F_{i}$ of each limb $l_{i}$ is defined as a line force passing through the center of the spherical joint $S_{i}$ along the direction parallel to the revolute joint $r_{i}$ [10] [17].

$$
F_{i}=\left[r_{i},\left(S_{i} \times r_{i}\right)\right]
$$

\section{Actuated wrench system}

When the actuated prismatic joint of $l_{i}$ is locked, only 4-system twist is valid. Consequently each prismatic actuated wrench, $W_{i}$, of each $l_{i}$ form 2-system wrench and only reciprocal to all passive 4-system twist $\hat{\$}_{r i}, \hat{\$}_{u i}, \hat{\$}_{v i}$ and $\hat{\$}_{w i}$. Then all reciprocal screws lie on a plane $\alpha_{i}$ (Figure 5) [10] [11]. When the actuated revolute joint of $l_{i}$ is locked, only 4-system twist is valid. Consequently each revolute actuated wrench, $W_{i}^{\prime}$, of each $l_{i}$ form 2 -system wrench and only reciprocal to all passive 4-system twist $\hat{\$}_{p i}, \hat{\$}_{u i}, \hat{\$}_{v i}$ and $\hat{\$}_{w i}$. Then all reciprocal screws lie on a plane $\beta_{i}$ (Figure 5) [10] [11]. Spherical joint correspond to the three intersecting and no-coplanar revolute joints, when each actuated spherical joint of each limb PRS is locked, only 2-system twist will be valid for each limb PRS. Consequently each spherical actuated wrench, $\bar{W}_{n}$, of each limb-PR $\underline{\underline{S}}$ form 4-system wrench and only reciprocal to all remain passive 2-system twist $\hat{\$}_{p i}$ and $\hat{\$}_{r i}[10][11]$

\section{Global Wrench System of 3-PRS PMs}

Each limb $l_{i}$ is identified by $F_{i}$ and $W_{i}$. Both graphical representation of $G W S$ is called wrench graph

For: 3-PRS (Figure 8)

$$
\begin{aligned}
& \operatorname{det}\left(J_{6 \times 6}\right)=G W S \\
& G W S=\left[\alpha_{1}, \alpha_{2}, \alpha_{3}, F_{1}, F_{2}, F_{3}\right] \text { with } F_{i} \in \alpha_{i}
\end{aligned}
$$

Each $F_{i}$ and $r_{i}$ are parallel, in projective geometry they intersect each over at infinity at a unique point. Then $b \equiv d, f \equiv h$ and $j \equiv l$ respectively for limbs $l_{1}, l_{2}$ and $l_{3}$. For notation convenience, let designed by the capital letters these points at infinity and rewrite Equation (15) as:

$$
G W S=[a B, c B, e F, g F, i J, k J] \text { and } P=a B \vee c B \vee e F \vee g F \vee i J \vee k J
$$

The Superbracket in Equation (16) is decomposed, reduced [12] and gave:

$$
\begin{aligned}
& {[a B, c B, e F, g F, i J, k J] } \\
= & +[a B c e][B g F J][F i k J]-[a B c F][B g F J][e i k J] \\
& -[a B c g][B e F J][F i k J]+[a B c F][B e F J][\text { gikJ }]
\end{aligned}
$$




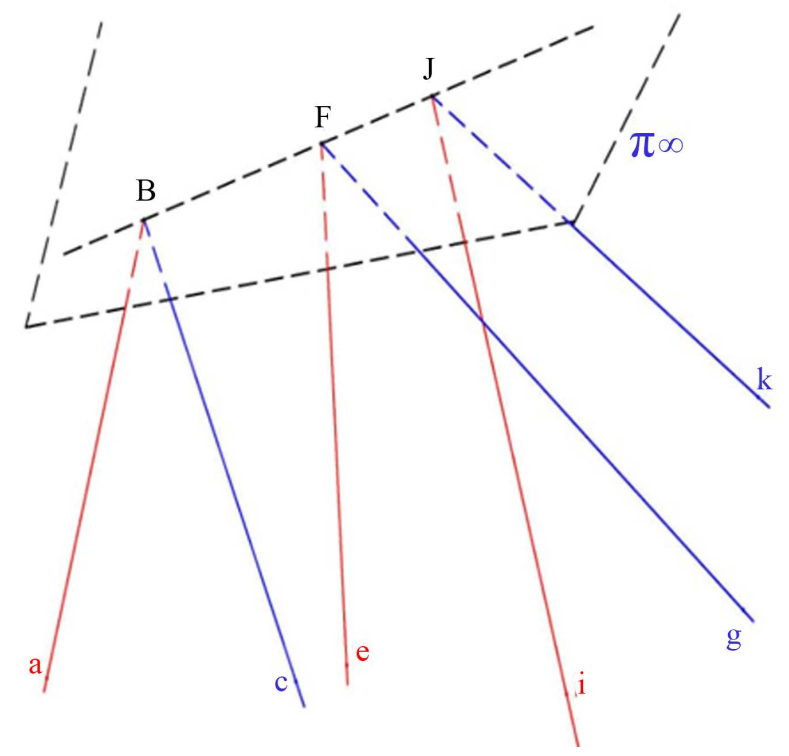

Figure 8. Wrench graphs of $\underline{\mathrm{P} R S}$.

Equation (17) vanishes at singularities configuration with the condition:

$$
[a B, c B, e F, g F, i J, k J]=0 \text { when }[x B F J]=0 \text { or } a B c \wedge i k J \wedge e F
$$

$x=\{a, b, c, e, g, i, k\}$, in singular configuration: $[x B F J]=0$ or $a B c \wedge i k J \wedge e F$

If $[a B F J]=0$ then $a B \wedge F J$ means $F$ collinear to $a B$ or $J$ collinear to $a B$. Since $F$ and $J$ are respectively the unique intersecting point at infinity of parallel lines $e F, g F$ and $i J, k J$ then $F$ collinear to $a B$ or $J$ collinear to $a B$ which means $a B$ intersect also $e F, g F, i J$ and $k J$ at $F$ as infinity point. Since two parallel lines intersect each over at a unique point at infinity it is obvious that $a B$ is parallel to $e F, g F, i J$ and $k J$. This means $F_{1}$ parallel to $F_{2} W_{2} F_{3} W_{3}$. Similarly if $[c B F J]=0$ with the same processes, $W_{1}$ Parallel to $F_{2}, W_{2}, F_{3}, W_{3}$. For $l_{1}$, the singularity occurs when $F_{1}$ or $W_{1}$ parallel to $F_{2}, F_{3}, W_{2}, W_{3}$. It is obvious to deduce for $l_{i}$. If $a B c \wedge i k J \wedge e F$ means the line $F_{2}$ crosses the intersection line of the two planes $\alpha_{1}$ and $\alpha_{3}$

For 3-PRS (Figure 9)

$$
\begin{aligned}
& \operatorname{det}\left(J_{6 \times 6}^{\prime}\right)=(G W S)^{\prime} \\
& (G W S)^{\prime}=\left[\beta_{1}, \beta_{2}, \beta_{3}, F_{1}, F_{2}, F_{3}\right] \text { with } F_{i} \in \beta_{i}
\end{aligned}
$$

Any line can be represented in projective space by one finite point and one point at infinity, let choose one point at infinity on each of these six Plücker lines. Equation (19) can be rewrite as:

$$
(G W S)^{\prime}=[A b, b N, E f, f P, I j, j R] \text { and } P^{\prime}=A b \vee b N \vee E f \vee f P \vee I j \vee j R
$$

Any line can be represented in projective space by one finite point and one point at infinity. Let choose one point at infinity on each of these six Plücker lines described as: $a \equiv A, n \equiv N, e \equiv E, p \equiv P, i \equiv I, r \equiv R$. Equation (20) can be rewritten as: 


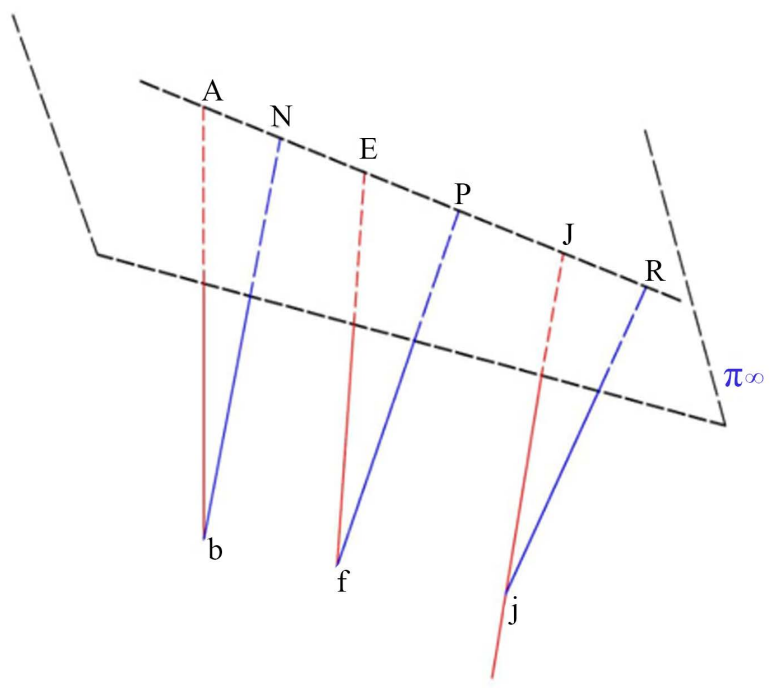

Figure 9. Wrench graph of 3-PRS PMs.

$$
(G W S)^{\prime}=[A b, b N, E f, f P, I j, j R] \text { and } P^{\prime}=A b \vee b N \vee E f \vee f P \vee I j \vee j R
$$

The Superbracket in Equation (21) decomposed and reduced with the given tool [12]:

$$
\begin{aligned}
{[} & A b, b N, E f, f P, I j, j R] \\
= & -[A b N E][b f P j][f I j R]+[A b N f][b f P j][E I j R] \\
& +[A b N f][b E f j][P I j R]-[A b N P][b E f j][f I j R]
\end{aligned}
$$

Equation (22) vanishes at singularities configuration with the condition: $[A b, b N, E f, f P, I j, j R]=0$ when $A b N \wedge b f j \wedge I j R \wedge E P f$ The singular configuration arises when the four planes $\beta_{1}, \beta_{2}, \beta_{3}$ and $b f j$ intersect at a unique point.

For 3-PRS

$$
\begin{aligned}
& \operatorname{det}\left(J_{6 \times 6}^{\prime \prime}\right)=(G W S)^{\prime \prime} \\
& (G W S)^{\prime \prime}=\left[\gamma_{1}, \gamma_{2}, \gamma_{3}, F_{1}, F_{2}, F_{3}\right] \text { with } \gamma_{i} \equiv\left(p_{i}, r_{i}\right)
\end{aligned}
$$

Instead of 6 Plücker coordinates lines we found nine Plücker coordinates lines. This means in such situation, the frame rigidity of the mechanism has problem. Once the spherical actuator is locked the number of Plücker lines increase to two-9 extensor. Equation (23) confirms this absurdity. When the actuated spherical joint is locked the number $\mathrm{n}$ of degree of freedom becomes negative then "it is preloaded structure and no motion is possible and some stresses may also be presented at the time of assembly" [19]. The tool provides by Stéphane Caro et al. [12] can neither decompose nor reduce it.

\subsection{Pure Condition Analysis in Grassmann-Cayley Approach}

The determinant of Jacobian matrix equal to zero or the superbracket vanishes. In this situation the serial robot controller will demand power from the actuators 
to meet infinity joint rate requirement and the actuators will blow off. The actuating prismatic joints reached robot in singularity configuration when the actuated force or the constraint force of any $l_{k}$ is parallel to either a constraint force or an actuated force of $l_{k^{\prime}}$ with $k \neq k^{\prime}$. Singular motion arises when the common actuated force of $l_{1}$ and $l_{3}$ crosses the constraint force of $l_{2}$. The revolute actuated joints reached robot in singularity configuration when the four planes $\beta_{1}, \beta_{2}, \beta_{3}$ and $b f j$ intersect at a unique point. The common actuated wrench of the three limbs crosses the plane formed by the three constraint forces at a point and then the robot manipulator is in a plane configuration. The spherical actuated joints give different form of the symbolic Plücker coordinates lines. Instead of 6 Plücker coordinates lines we found nine Plücker coordinates lines. Once the spherical actuator is locked the number of Plücker lines increase to two-9 extensor. Equation (23) confirms this absurdity. The results presented above show that firstly whatever wrist singularity is theoretical possible; practically this singularity could be possible if only if the design of two of the last three axis are parallel. Secondly, when the spherical joint is actuated, the architecture mechanism becomes preload and stressed. This suggests that the rigidity of the manipulators depend of the position of the actuated joints in the mechanism. Thirdly, parallel robot or serial robot, the six legs bars have dependent Plücker coordinates when the architecture lacks rigidity in the critical pose. And the last but not the least, we suggested that the rigidity framework of the robots manipulators depends on both architecture structure and operating mode.

\section{Conclusion}

This paper presented the geometric interpretation of pure condition which means simultaneous singularity and rigidity analysis on both 6R SMs through a variable orientation joint and 3-PRS PMs within variable actuated joints with no coordinate approach based on GCA. Symbolic TS and GWS were respectively applied on serial and parallel robots to determine the vanished points (singularity) of their superbrackets. Existing theories suggested that GCA should only apply for the singularity of parallel robots; the results, however, show that serial parallel robots can be investigated using GCA. This evidence let us to remark that parallel robot is combination of some serial kinematic chains. Based on the discussion in the preceding sections, the following conclusions can be drawn: For 6R SMs, singularity of the serial shoulder and elbow singularities arose. It was deduced that wrist singularity for $6 \mathrm{R}$ SMs could be possible if and only if the design of two of the last three axes were parallel according to the orientation of the actuators. The rigidity of the framework is dependent of the operating mode of the architecture. For 3-PRS PMs, where each fully kinematic chain had only one actuator, singularity arose when three constraints forces lie on a common plane and intersect at the particular point. The negative value for the DOF of the mechanism of locked and actuated spherical joint confirmed that the rigidity of the framework is dependent of the position of the actuated join and the operat- 
ing mode of the architecture. We recognize that the method adopted in current study does not cover all class of robot such as hybrid robot, hybrid parallel robot which will be our future research in the next paper. These results suggest that pure condition confirmed that both position and orientation of the actuator have to be integrated in the conceptual design stage in order to avoid singularities configuration and to optimize the rigidity frame of the architecture. The analysis of robot pure condition is a practical problem of robot manipulators designers who need many mathematical theories such as Geometry Algebra, Lie Group theory; therefore Robotic field is a multidisciplinary field belongs to Mechatronics applications.

\section{Acknowledgements}

I would thank Dr. (MC) Florent QUENUM, for his advice and encouragement during my research. I'm also grateful to Professor Stéphane CARO, to provide his graphic user interface for superbrackets computation.

\section{Conflicts of Interest}

The authors declare no conflicts of interest regarding the publication of this paper.

\section{References}

[1] Downing, D.M., Samuel, A.E. and Hunt, K.H. (2002) Identification of the Special Configurations of the Octahedral manipulator Using the Pure Condition. The International Journal of Robotics Research, 21, 147. https://doi.org/10.1177/027836402760475351

[2] Hayes, M.J.D., Husty, M.L. and Zsombor-Murray, P.J. (2002) Singular Configurations of Wrist-Partitioned 6R Serial Robots: A Geometric Perspective for Users. Transactions of the Canadian Society for Mechanical Engineering, 26, 41-55. https://doi.org/10.1139/tcsme-2002-0003

[3] Chen, Y.-C. and Chen, C.L.P. (1994) An Analysis to the Singularity of Serial Manipulator Using Reciprocal Screw Theory. IEEE International Conference on Systems, Man, and Cybernetics, Humans, Information and Technology, 1, 148-153.

[4] Boudreau, R. and Podhorodeski, R.P. (2010) Singularity Analysis of a Kinematically Simple Class of 7 Jointed Revolute Manipulators. Transactions of the Canadian Society for Mechanical Engineering, 34, 105-117.

[5] Carretero, J.A., Podhorodeski, R.P., Nahon, M.A. and Gosselin, C.M. (2000) Kinematic Analysis and Optimization of a New Three Degree-of-Freedom Spatial Parallel Manipulator. ASME. Journal of Mechanical Design, 122, 17-24.

[6] Tchoń, K. and Muszysńki, R. (1998) Singular Inverse Kinematic Problem for Robotic Manipulators: A Normal Form Approach. IEEE Transactions on Robotics and Automation, 14, 93-104. https://doi.org/10.1109/70.660848

[7] Rocha, D.M. and da Silva, M.M. (2013) Workspace and Singularity Analysis of Redundantly Actuated Planar Parallel Kinematic Machines. Proceedings of the XV International Symposium on Dynamic Problems of Mechanics.

[8] Husty, M., Ottaviano, E. and Ceccarelli, M. (2008) Advances in Robot Kinematics: 
Analysis and Design. A Geometrical Characterization of Workspace Singularities in 3R Manipulators. Springer Netherlands, 411-418.

[9] Selvakumar, A.A., Babu, R.P. and Sivaramakrishnan, R. (2012) Simulation and Singularity Analysis of 3-PRS Parallel Manipulator. Proceedings of IEEE, International Conference on Mechatronics and Automation, 2203-2207.

[10] Kanaan, D., Wenger, P., Caro, S. and Chablat, D. (2009) Singularity Analysis of Lower Mobility Parallel Manipulators Using Grassmann-Cayley Algebra. IEEE Transactions on Robotics, 25, 995-1004. https://doi.org/10.1109/TRO.2009.2017132

[11] Amine, S., Tale-Masouleh, M., Caro, S., Wenger, P. and Gosselin, C. (2011) Singularity Analysis of the 4-RUU Parallel Manipulator using Grassmann-Cayley Algebra. CCToMM Symposium on Mechanisms, Machines and Mechatronics, Montreal.

[12] Amine, S. and Caro, S. Singularity Conditions of Lower-Mobility Parallel Manipulators Based on Grassmann-Cayley Algebra. L'Agence nationale de la recherche. http://www.irccyn.ec-nantes.fr/ caro/SIROPA/GUIGCASiropa.jar

[13] Arvin, R. and Mehdi Tale, M. Singularity Configurations Analysis of a 4-DOF Parallel Mechanism Using Grassmann-Cayley Algebra.

[14] Ben-Horin, P. and Shoham, M. (2009) Application of Grassmann-Cayley Algebra to Geometrical Interpretation of Parallel Robot Singularities. The International Journal of Robotics Research, 28, 127-141. https://doi.org/10.1177/0278364908095918

[15] Amine, S., Masouleh, M.T., Caro, S., Wenger, P. and Gosselin, C. (2012) Singularity Conditions of 3T1R Parallel Manipulators with Identical Limb Structures Semaan. ASME Journal of Mechanisms and Robotics, 4, 1.

[16] Wen, K., Won Seo, T., et al. (2015) A Geometric Approach for Singularity Analysis of 3-DOF Planar Parallel Manipulators Using Grassman-Cayley Algebra. Cambridge University Press, Robotica, 1-10.

[17] Amine, S., Caro, S., Wenger, P. and Kanaan, D. (2012) Singularity Analysis of the H4 Robot Using Grassmann-Cayley Algebra. Robotica, 1-10.

[18] Staffetti, E. and Thomas, F. (2000) Kinestatic Analysis of Serial and Parallel Robot Manipulators Using Grassman-Cayley Algebra. In: Lenarcic, J. and Stanisic, M.M., Eds., Advances in Robot Kinematics, Springer Netherlands, 17-27. https://doi.org/10.1007/978-94-011-4120-8_2

[19] Norton, R.L. (2001) Design of Machinery. An Introduction to the Synthesis and Analysis of Mechanism and Machines. 2 Edition, McGraw-Hill Series in Mechanical Engineering, 32. 


\section{Nomenclatures}

\begin{tabular}{|c|c|}
\hline Symbols & nomenclatures \\
\hline$\vee:$ & Join operator (in Grassmann-Cayley Algebra) \\
\hline$\wedge:$ & Meet operator (in Grassmann-Cayley Algebra) \\
\hline$\cap:$ & Intersection of vectors \\
\hline$\oplus:$ & Spanning by vectors \\
\hline$F_{i}:$ & Constraint wrench force of the $i^{\text {th }}$ limb for the Parallel Robot \\
\hline$\sigma:$ & Permutation \\
\hline$\rho:$ & Wrench intensity \\
\hline$\Gamma_{p, q}:$ & $\begin{array}{l}\text { Plücker coordinate vector of finite line passing through two distinct finite } \\
\text { points } \mathrm{p} \text { and } \mathrm{q}\end{array}$ \\
\hline$\Gamma_{\infty}:$ & $\begin{array}{l}\text { Plücker coordinate vector of lines at infinity, passing through two points at } \\
\text { infinity }\end{array}$ \\
\hline$J_{6 \times 6}:$ & Jacobian of Square Matrix of six columns six rows \\
\hline$k$ & Number of joint \\
\hline$l_{i}:$ & $I^{\text {th }}$ Limb or $I^{\text {th }}$ kinematic chain \\
\hline$m:$ & Number of link \\
\hline$\eta:$ & Order of task space \\
\hline$P^{3}:$ & Projective Space of 3 dimensional \\
\hline$P_{s}:$ & Symbolic level of Plücker coordinates \\
\hline$p_{i}:$ & Prismatic joint axis of $I^{\text {th }} \operatorname{limb}$ \\
\hline$\pi_{\infty}:$ & Plane at infinity in the Projective Space \\
\hline$r_{i}:$ & Revolute joint axis of the $I^{\text {th }}$ limb \\
\hline$s_{i}:$ & Spherical joint axis of the $I^{\text {th }}$ limb \\
\hline$S_{0}:$ & Position Vector of any point on the screw axe \\
\hline$S$ & Unit vector along the screw axis \\
\hline$\hat{\$}_{0}:$ & Zero pitch screw \\
\hline$\hat{\$}_{\infty}:$ & Infinite pitch screw \\
\hline$V:$ & Vector Space \\
\hline$W_{n}:$ & $n^{\text {th }}$ Actuated wrench force \\
\hline$\hat{\$}_{r 0}:$ & Wrench of zero pitch \\
\hline$\hat{\$}_{r \infty}:$ & Wrench of infinite pitch \\
\hline$[\underbrace{a, \cdots, b}_{n \text {-points }}]$ : & Superbracket which consist of $\mathrm{n}$ points \\
\hline$S_{0} \times s:$ & Moment of the screw related to the origin of the reference frame \\
\hline
\end{tabular}

